\title{
DATA ANALYTICS APPROACH TO PREDICT THE HARDNESS OF COPPER MATRIX COMPOSITES
}

\author{
Somesh Kr. Bhattacharya ${ }^{1}$, Ryoji Sahara ${ }^{l}$, Dušan Božić ${ }^{2}$ Jovana Ružić ${ }^{2, *}$ \\ ${ }^{I}$ Research Center for Structural Materials, National Institute for Materials Science, \\ 1-2-1 Sengen, Tsukuba, Ibaraki 305-0047, Japan \\ ${ }^{2}$ Department of Materials, "VINČA" Institute of Nuclear Sciences - National Institute of \\ the Republic of Serbia, University of Belgrade, PO Box 522, 11001 Belgrade, Serbia
}

Received 25.09.2020

Accepted 22.10.2020

\begin{abstract}
Copper matrix composite materials have exhibited a high potential in applications where excellent conductivity and mechanical properties are required. In this study, the machine learning models have been applied to predict the hardness of the copper matrix composite materials (CuMCs) produced via the powder metallurgy technique. Six different machine learning regression models were employed. The observed CuMCs were reinforced with two different volume fractions ( 2 vol. $\%$ and $7 \mathrm{vol} . \%)$ of $\mathrm{ZrB}_{2}$ particles. Based on experimental work, we extracted the independent variables (features) like the milling time (MT, Hours), dislocation density (DD, $\mathrm{m}^{-2}$ ), average particle size (PS, $\mu \mathrm{m})$, density $\left(\rho, \mathrm{g} / \mathrm{cm}^{3}\right)$ and yield stress $(\sigma, \mathrm{MPa})$ while the Vickers hardness ( $\mathrm{MPa})$ was used as the dependent variable. Feature selection was performed by calculation the Pearson correlation coefficient (PCC) between the independent and dependent variables. The predictive accuracy higher than $80 \%$ was achieved for $\mathrm{Cu}-7 \mathrm{vol} . \% \mathrm{ZrB}_{2}$ and lower for the $\mathrm{Cu}-2 \mathrm{vol} . \% \mathrm{ZrB}_{2}$.
\end{abstract}

Keywords: Copper Matrix Composites; Hardness; Machine Learning; Regression Model.

\section{Introduction}

The excellent electrical conductivity and mechanical properties of copper matrix composites (CuMCs) and copper alloys [1, 2, 3] make them desirable materials in several industries viz. automotive, aerospace, military, nuclear, electronic. The main potential of these materials lies in reaching the favorable relationship between improving the mechanical properties and preserving high conductivity. It is well known that the lower content of alloying elements in the copper matrix supports higher thermal

${ }^{*}$ Corresponding author: Jovana Ružić, jovana.ruzic@gmail.com 
and electrical conductivity. The most commonly used reinforcements $[4,5]$ for the copper matrix are metals ( $\mathrm{Ti}, \mathrm{Mg}, \mathrm{Co}, \mathrm{Ni}$, etc.) or ceramic particles $\mathrm{SiC}$ and $\mathrm{Al}_{2} \mathrm{O}_{3}$, while in recent years, particles such as $\mathrm{ZrB}_{2}, \mathrm{TiO}_{2}, \mathrm{TiB}_{2}, \mathrm{TiC}, \mathrm{B}_{4} \mathrm{C}$, etc., are used. Since the properties of the CuMCs and copper alloys strongly depend on the nature, amount, and distribution of the reinforcements, great attention is given to the selection of the manufacturing techniques for their production. Ingot and powder metallurgy are both used for the production of the $\mathrm{Cu}$-based materials, where powder metallurgy is more suitable when in situ formation of the reinforcing particles is needed $[6,7,8,9,10]$. Although, the most recent study [11] of the copper matrix particulate-reinforced composite material, where $\mathrm{ZrB}_{2}$ ceramics is used as reinforcement, produced by ingot metallurgy show that as-cast $\mathrm{Cu}-\mathrm{ZrB}_{2}$ composites can reach the improvement in hardness up to 140 Vickers Hardness (HV) similar to the results obtained by powder metallurgy [12]. According to the facts mentioned above, the copper-based materials' investigation attracts both researchers and engineers from different fields due to their wide application and fast industry growth.

In the powder metallurgy technique, the properties of alloys and metal matrix composites (MMCs) depend mainly on the milling time. Thus, it is highly desirable to have a rapid and accurate prediction of the hardness via the structure-property correlation of these MMCs. The physics-based models (e.g., density functional theory and phase field simulations) can promote understanding at a given length scale. Still, they are often limited to low order model systems due to computational complexity and lack of input parameters to represent realistic higher-order systems. An efficient way to achieve the mechanical properties' prediction is the data-driven methodology that involves applying statistical learning tools to analyze correlations between hardness and features of the MMCs. The machine learning (ML) approach can reduce the experimental cost and time while predicting materials' target properties [13, 14, 15, 16].

In the present study, we made an attempt to apply ML approach to predict the hardness of the CuMCs. To predict the materials' hardness, six different regression models were employed: random forest regression, gradient boosting regression, support vector regression, k-Nearest neighbors regression, linear regression, and kernel ridge regression. The predictive accuracy of the applied models was discussed.

\section{Methodology}

\section{Experimental work}

The $\mathrm{Cu}-\mathrm{ZrB}_{2}$ alloy was produced using the powder metallurgy technique, where $\mathrm{Cu}, \mathrm{Zr}$, and $\mathrm{B}$ were used as starting powders. Mechanical alloying was performed in the attritor mill. The in situ formation of $\mathrm{ZrB}_{2}$ particles inside the $\mathrm{Cu}$ matrix was achieved during hot-pressing at $950^{\circ} \mathrm{C}$. The amount of reinforcing $\mathrm{ZrB}_{2}$ particles was set as $2 \mathrm{vol} . \%$ and $7 \mathrm{vol} \%$ in the $\mathrm{Cu}$ matrix. Morphological analyses of the mechanically alloyed (MA) powder mixtures were done by particle sizer and scanning electron microscopy (SEM). Microstructural characterization of the MA powder mixtures and hot-pressed samples were characterized by X-ray powder diffraction (XRD) and SEM. The detailed production procedure of $\mathrm{Cu}-\mathrm{ZrB}_{2}$ composites and characterization methods applied have been described in previous studies [12, 17, 18]. 


\section{Machine learning models}

The primary requirement to build a statistical learning model for any material is to have a dataset containing the material descriptors or features, $\mathrm{X}$. These descriptors represent the fundamental material properties. The basic task of the machine learning (ML) models if to map these features to a specific (target) property, $Y$ (hardness in this case), that is $\boldsymbol{Y}=\boldsymbol{f} \boldsymbol{X}$. Thus, the two important elements of the machine learning approach are the empirical model, $f$, and features, $\boldsymbol{X}$. The ML model must be trained and cross-validated using the training dataset, which includes the measured targeted property. The trained model is then applied to an unseen dataset in order to predict the target property. From the experiments, milling time (MT, Hours), dislocation density $\left(\mathrm{DD}, \mathrm{m}^{-2}\right)$, average particle size (PS, $\left.\mu m\right)$, density $\left(\rho, \frac{\mathrm{gm}}{\mathrm{cm}^{3}}\right)$ and yield stress $(\sigma, \mathrm{MPa})$ as our descriptors, $\boldsymbol{X}$, were selected. It is well known that the final mechanical properties of any material strongly depend on its process parameters. As powder metallurgy is a time-consuming process, the establishment of such correlations through experiments takes time measured in months and very often in years. Hence, the ML models were used to support and accelerate research in this field. In this study, two datasets for two different CuMCs are used: (i) $\mathrm{Cu}-7 \mathrm{vol} . \% \mathrm{ZrB}_{2}$ and (ii) $\mathrm{Cu}-2 \mathrm{vol} . \% \mathrm{ZrB}_{2}$. Datasets consist of only sixteen observations (data points) for $\mathrm{Cu}-7 \mathrm{vol} . \% \mathrm{ZrB}_{2}$ and twelve for $\mathrm{Cu}-2 \mathrm{vol} . \% \mathrm{ZrB}_{2}$. As described later, a special fitting method has been applied to improve the machine learning model performance and achieved a reasonable predictive model. In both cases, the datasets are found to be small compering to literature. On the other hand, the presented results show that these small datasets are enough to understand the trend for these mechanically alloyed CuMCs powders considered in the present study.

To predict the hardness of both materials, $\mathrm{Cu}-7 \mathrm{vol} . \% \mathrm{ZrB}_{2}$ and $\mathrm{Cu}-2 \mathrm{vol} . \% \mathrm{ZrB}_{2}$, different ML models were used: random forest (RF) regression [19], gradient boosting (GB) regression [20], support vector (SV) regression [21], k-Nearest neighbors (KNN) regression [22], linear regression (LR) [23] and kernel ridge (KR) regression [24] as implemented in the Python-based open-source data analytics toolkit, scikit-learn [25]. $\mathrm{RF}$ and GB regression models are ensemble learning methods where multiple decision trees are constructed. SV regression is considered a nonparametric technique as it relies on kernel functions. The linear regression models the relationship between the input and output variables using a linear predictor function and fits to minimize the residual sum of squares between observed data and predicted data. Kernel ridge regression estimates the conditional expectation of a random variable to find a non-linear relationship between a pair of random variables. Using the kernel method, it simplifies the product of the inner products in a high dimensional space and learns a linear model in the implicit feature space induced by the kernel and the dataset. k-Nearest neighbors regression model uses a nonparametric method and outputs the average number of given data points, the $\mathrm{k}$ nearest neighbors. Due to the availability of the small dataset, Leave One Out (LOO) - cross validation (CV) was performed [26]. The training of ML models with $\mathrm{CV}$ avoids the errors due the bias and variance. Finally, the hyperparameters for the ML models were optimized during the training process. For model performances, the coefficient of determination, $R^{2}$, was calculated [27]. It is important to note that for both these CuMCs, the ML models were trained separately with their respective datasets. 


\section{Results and discussion}

A strong influence of the milling parameters on the morphological and mechanical properties of alloys and MMCs has been reported in many studies. The duration of the milling process is essential in providing uniform distribution of the reinforcing particles in the metal matrix. During milling in the Attritor mill, the powder mixture is exposed to high energy collisions such as ball-particle-ball and wall-particleball. Those collisions initiate changes in lattice parameters, shape, and size, as well as the hardness of the particles. Finding the suitable milling parameters for each alloy or composite material is a time-consuming process. According to all mentioned-above, and considering relations between process parameters and changes in powder properties, the input parameters for ML models were selected.

In this study, first, all the features were subjected to the correlation filter to remove those who are uncorrelated by calculating the Pearson correlation coefficient (PCC). The Pearson correlation is the measure of the linear correlation between the predictors, $\boldsymbol{X}$, and target, $\boldsymbol{Y}$. The Pearson correlation maps for both the CuMCs are shown in Fig. 1. For both the MCs, we observed the yield stress and density to have the strongest correlation with hardness, followed by dislocation density. In the case of $\mathrm{Cu}$ 2vol.\% $\mathrm{ZrB}_{2}$ composite (see Fig. 1(a)), milling time, and particle size were found to have a negative correlation. While the milling time was found to have a negligible correlation with hardness, the particle size was found to have a weak negative correlation. The particle size was found to have the lowest correlation coefficient in the case of $\mathrm{Cu}-7 \mathrm{vol} . \% \mathrm{ZrB}_{2}$ composite. Importantly, all the features were found to have a positive correlation coefficient for $\mathrm{Cu}-7 \mathrm{vol} . \% \mathrm{ZrB}_{2}$ composite. All the features were found to have positive correlation coefficients for $\mathrm{Cu}-7 \mathrm{vol} . \% \mathrm{ZrB}_{2}$ composite. It should be noted that all the features of $\mathrm{Cu}-7 \%$.vol $\mathrm{ZrB}_{2}$ composite are used for fitting the ML models but in the case of $\mathrm{Cu}-2 \mathrm{vol} . \% \mathrm{ZrB}_{2}$ composite, the feature "milling time" was dropped.
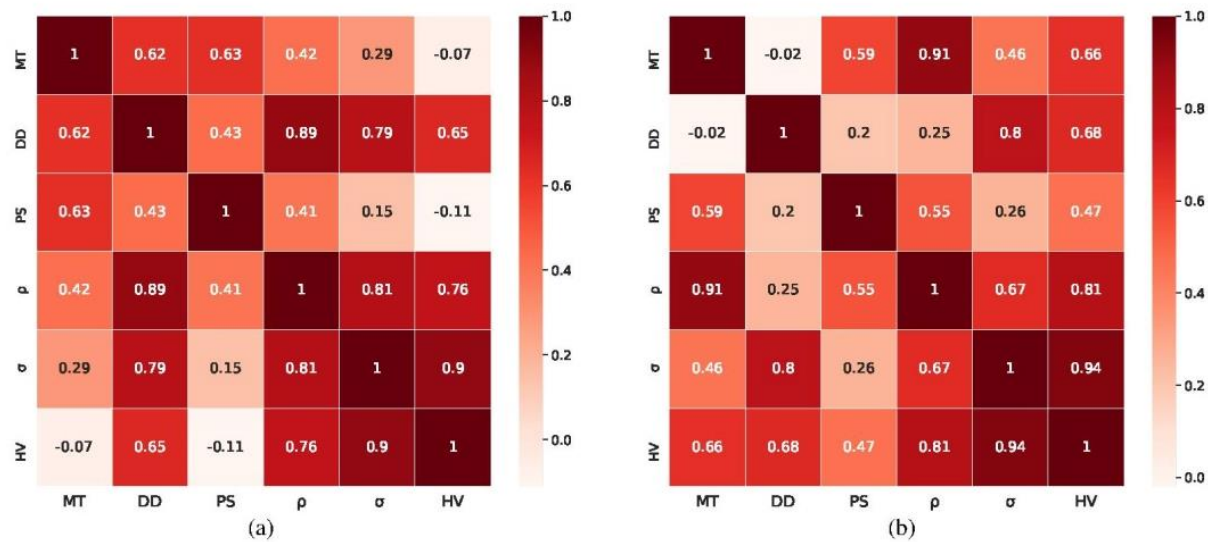

Fig. 1. The Pearson correlation maps for the features and the target for (a) $\mathrm{Cu}-2 \mathrm{vol} . \%$ $\mathrm{ZrB}_{2}$, and (b) $\mathrm{Cu}-7 \mathrm{vol} . \% \mathrm{ZrB}_{2}$. The color tone depicts the significance of the correlation. 
Next, the ML models were trained using LOO-CV, and the coefficient of determination $\left(R^{2}\right)$ was calculated to evaluate the model performance. The coefficient of determination $\left(R^{2}\right)$ which is calculated as

$$
R^{2}=1-\frac{\sum_{i=1}^{n}\left(y_{i}-\hat{y}_{i}\right)}{\sum_{i=1}^{n}\left(y_{i}-\bar{y}\right)}
$$

where $y_{i}$ is the true value, $\hat{y}_{i}$ is the predicted value and $\bar{y}$ is the mean of $y_{i}$. The $R^{2}$ the value lies between 0 and 1 , with 1 signifying excellent fits.

The considered value range is the one experimentally obtained. Experimental results showed that hardness increases up to 160 of Vickers hardness (maximum achieved at 25 hours of mechanical alloying) then decreases with increasing time of mechanical alloying. Vickers hardness values below 60 were not taken into consideration since pure copper shows Vickers hardness values higher than 60. In Table 1 , the $R^{2}$ for $\mathrm{Cu}-7 \mathrm{vol} . \% \mathrm{ZrB}_{2}$ composite are summarized. In this case, the random forest and kernel ridge regressor models exhibited the highest accuracy $(92 \%)$ followed by gradient boosting regressor $(88 \%)$ while the nearest neighbor regressor has the lowest accuracy of $79 \%$. It is evident that all the models were able to achieve an accuracy of $80 \%$ or even higher. For the two best performing ML models, random forest and kernel ridge, the true and predicted values of hardness for the $\mathrm{Cu}-7 \mathrm{vol} . \% \mathrm{ZrB}_{2}$ composite were plotted, as shown in Fig. 2.

Table 1. The coefficient of determination $\left(R^{2}\right)$ and mean absolute error (MAE) values obtained for the various $\mathrm{ML}$ methods applied to the $\mathrm{Cu}-7$ vol.\% $\mathrm{ZrB}_{2}$ composite are listed below.

\begin{tabular}{ccccccc}
\hline $\begin{array}{l}\text { ML } \\
\text { models }\end{array}$ & RF & KR & GB & SV & LR & KNN \\
\hline$R^{2}$ & 0.92 & 0.92 & 0.88 & 0.84 & 0.83 & 0.79 \\
\hline
\end{tabular}
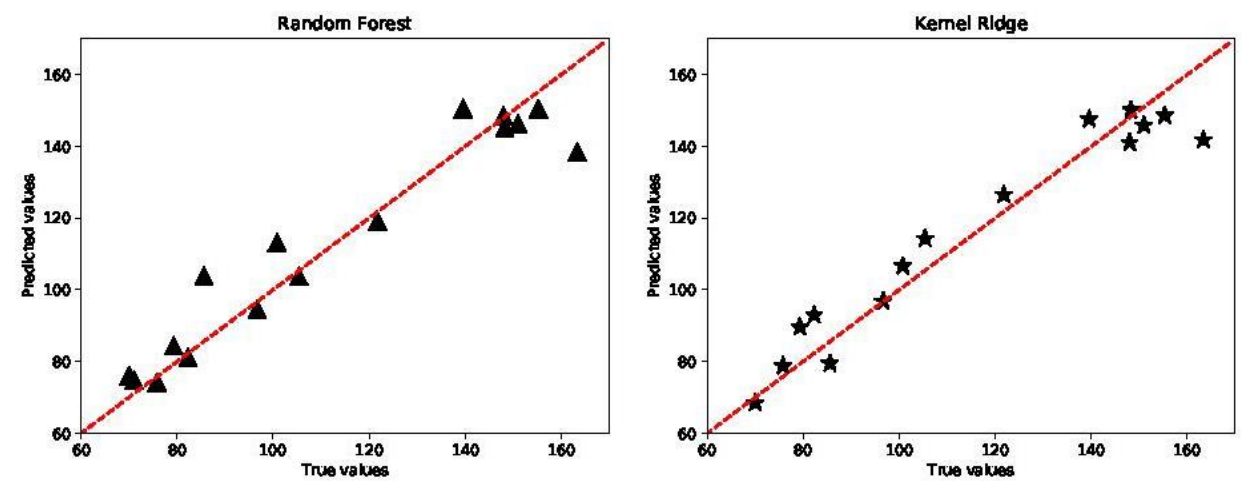

Fig. 2. The plots for the true values of Vickers hardness (experiment) and the predicted values of hardness using the two best performing $\mathrm{ML}$ models for $\mathrm{Cu}-7 \mathrm{vol} . \% \mathrm{ZrB}_{2}$ 
composite are plotted. The broken red line depicts the case where the true and predicted values exactly match.

The $R^{2}$ values obtained for the different ML models applied to $\mathrm{Cu}-2 \mathrm{vol} . \% \mathrm{ZrB}_{2}$ composite are shown in Table 2. For gradient boosting, an accuracy of $79 \%$ was achieved while for the support vector regressor and for kernel ridge regressor an accuracy of $74 \%$ was reached. Overall, all the ML models have lower accuracy in the case of $\mathrm{Cu}-2 \mathrm{vol} . \% \mathrm{ZrB}_{2}$ compared to $\mathrm{Cu}-7 \mathrm{vol} . \% \mathrm{ZrB}_{2}$ composite. It can be assumed that more data, leading to a larger dataset, is necessary to make a better predictive model for the hardness of $\mathrm{Cu}-2 \mathrm{vol} . \% \mathrm{ZrB}_{2}$ composite. In Fig. 3., the true and predicted values of hardness for the $\mathrm{Cu}-2 \mathrm{vol} . \% \mathrm{ZrB}_{2}$ composite for the gradient boosting and random forest models are plotted. Overall, all the ML models have lower accuracy in the case of $\mathrm{Cu}$ $2 \mathrm{vol} . \% \mathrm{ZrB}_{2}$ compared to $\mathrm{Cu}-7 \mathrm{vol} . \% \mathrm{ZrB}_{2}$ composite. An increase in the size of the data set will definitely increase the predictive power of the ML model for the hardness of $\mathrm{Cu}-\mathrm{ZrB}_{2}$ composite system.

Table 2. The coefficient of determination $\left(R^{2}\right)$ and mean absolute error (MAE) values obtained for the various $M L$ methods applied to the $\mathrm{Cu}-2$ vol.\% $\mathrm{ZrB}_{2}$ composite are listed below.

\begin{tabular}{lllllll}
\hline ML models & SV & KR & LR & GB & RF & KNN \\
\hline$R^{2}$ & 0.79 & 0.74 & 0.68 & 0.62 & 59 & 0.50 \\
\hline
\end{tabular}
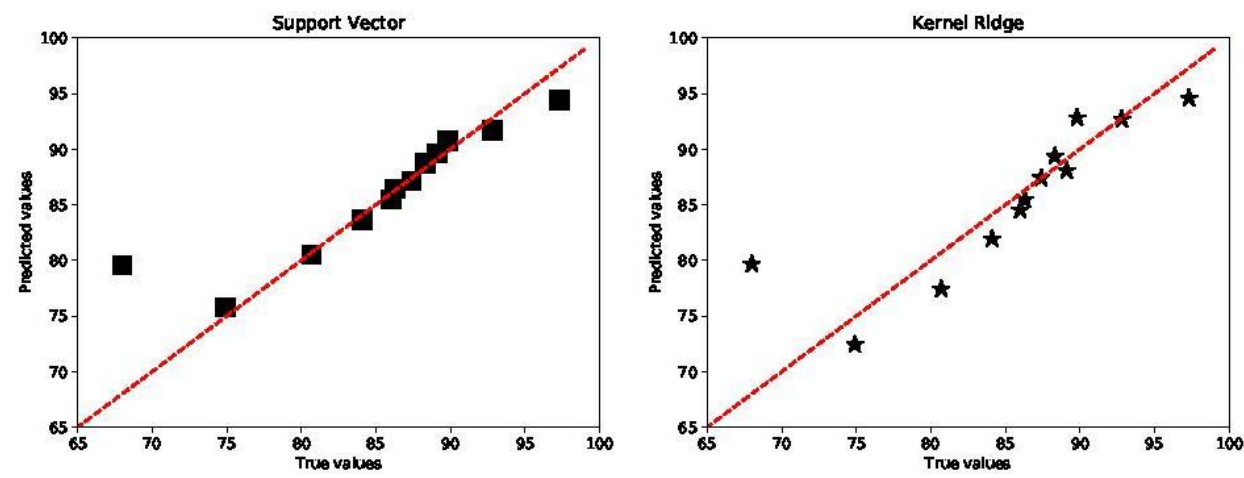

Fig. 3. The plots for the true values of Vickers hardness (experiment) and the predicted values of hardness using the two best performing $\mathrm{ML}$ models for $\mathrm{Cu}-2 \mathrm{vol} . \% \mathrm{ZrB}_{2}$ composite. The broken red line depicts the case where the true and predicted values exactly match.

\section{Conclusion}

In summary, a regression model was built to predict the hardness of CuMCs prepared by the powdered milling method. For $\mathrm{Cu}-7 \mathrm{vol} . \% \mathrm{ZrB}_{2}$ composite, an accuracy of $80 \%$ or higher was achieved. On the other hand, the ML models for $\mathrm{Cu}-2 \mathrm{vol} . \% \mathrm{ZrB}_{2}$ composite have a predictive accuracy lower than $79 \%$. To improve the accuracy of the ML models, additional data points must be included in the training dataset. A similar strategy can be extended to other matrix composites prepared by the mechanical alloying method and powder metallurgy technique. 


\section{Acknowledgments}

Authors, Somesh Kr. Bhattacharya and Ryoji Sahara, acknowledge the support from the project Council for Science, Technology and Innovation (CSTI), Crossministerial Strategic Innovation Promotion Program (SIP), "Materials Integration for revolutionary design system of structural materials" (Funding agency: JST). Authors, Dušan Božić, and Jovana Ružić acknowledge the support from the Ministry of Education, Science and Technological Development of the Republic of Serbia.

\section{References}

[1] K. U. Kainer, Metal matrix composites custom-made materials for automotive and aerospace engineering, WILEY-VCH Verlag GmbH \& Co. KGaA, Weinheim, 2006

[2] S. R. Pogson, P. Fox, C. Sutclisse, W. O'Neill: PRB 9 (2003) 334343.

[3] M. Li, S. J. Zinkle, Comprehensive nuclear materials 4 (2012) 667-690.

[4] Y. Zhan, Y. Z. J. Zeng: Tribol Lett 20 (2005) 163170.

[5] M. Sobhani, A. Mirhabibi, H. Arabi, R. M. D. Brydson: Mater Sci Eng A 577 (2013) 1622.

[6] W. S. Miller, F. J. Humphreys: Scripta Metallurgica et, 25 (1991) 33-38.

[7] C. Zou, Z. Chen, E. Guo, H. Kang, G. Fan, W. Wang: RSC Adv, 8 (2018) 30777-30782.

[8] S. J. Dong, Y. Zhou, Y. Shi, B. Chang: Metall Mater Trans A, 33(4) (2002) $1275-1280$.

[9] J. R. Groza, J. C. Gibeling: Mat Sci Eng A-Struct, 171 (1-2) (1993) 115-125.

[10] H. Kimura, N.Muramatsu, K.Suzuki, Copper alloy and copper alloy manufacturing method, Patent Application Publication, US 0211346A1, United States.

[11] X. Fan, X. Huang, Q. Liu, H. Ding, H. Wang, C. Hao: Results Phys, 14 (2019) $102494,1-6$.

[12] J. Ruzic, J. Stasic, S. Markovic, K. Raic, D. Bozic: Sci Sinter, 46 (2) (2014) 217224.

[13] D. Xue, D. Xue, R. Yuan, Y. Zhou, P. V. Balachandran, X. Ding, J. Sun, T. Lookman: Acta Materialia 125 (2017) $532-541$.

[14] D. Shin, Y. Yamamoto, M. Brady, S. Lee, J. Haynes, Modern data analytics approach to predict creep of high temperature alloys, Acta Mater, 168 (2019) 321-330.

[15] J. Wei, X. Chu, X.-Y. Sun, K. Xu, H.-X. Deng, J. Chen, Z. Wei, M. Lei: InfoMat 1 (3) (2019) 338-358.

[16] J. Schmidt, M. R. G. Marques, S. Botti, M. A. L. Marques: NPJ Comput Mater, 5 (2019) 83, 1-36.

[17] J. Ruzic, J. Stasic, V. Rajkovic, D. Bozic: Mater and Des, 62 (2014) 409-415.

[18] J. Ruzic, J. Stasic, V. Rajkovic, K. Raic, D. Bozic: Sci Eng Compos Mater, 22 (2015) 665-671.

[19] M. R. Segal, UCSF: Center for Bioinformatics and Molecular Biostatistics, (2004) 1-14.

[20] L. Mason, J. Baxter, P. Bartlett, M. Frean: Advances in neural information processing systems, MIT Press. 12 (1999) 512-518.

[21] H. Drucker, C. C. Burges, L. Kaufman, A. J. Smola, V. N. Vapnik: Advances in neural information processing systems, NIPS, MIT-Press, 9 (1997) 155-161. 
[22] V. Cherkassky, Y. Ma: Neural Comput, 15 (2003) 1691-1714.

[23] J. Neter, M. H. Kutner, C. J. Nachtsheim, W. Wasserman, Applied linear statistical models, 4 Edition, Chicago: Irwin, 1996

[24] Y. Zhang, J. Duchi, M. Wainwright: J Mach Learn Res, 16 (2015) 3299-3340.

[25] F. Pedregosa, G. Varoquaux, A. Gramfort, V. Michel, B. Thirion, O. Grisel, M. Blondel, G. Louppe, P. Prettenhofer, R. Weiss, V. Dubourg, J. Vanderplas, A. Passos, D. Cournapeau, M. Brucher, M. Perror, E. Duchesnay: J Mach Learn Res, 12 (2012) 2825-2830.

[26] C. Sammut, G. I. Webb, Leave-one-out cross-validation in encyclopedia of machine learning, Springer, Boston, MA, 2010.

[27] J. L. Devore, Probability and statistics for engineering and the sciences (8th ed.), Boston, MA: Cengage Learning (2011) 508-510.

\section{(C) (7) Creative Commons License}

This work is licensed under a Creative Commons Attribution 4.0 International License. 